\title{
Improving the Transglycosylation Activity of a- glucosidase From Xanthomonas Campestris Through Semi-rational Design for the Synthesis of Ethyl Vanillin-a-glucoside
}

\section{Luyi Chen}

Zhejiang University of Technology Chaohui Campus: Zhejiang University of Technology

Yi Liu

Zhejiang University of Technology Chaohui Campus: Zhejiang University of Technology

Yaoyao Zhou

Zhejiang University of Technology Chaohui Campus: Zhejiang University of Technology

Linjiang Zhu

Zhejiang University of Technology Chaohui Campus: Zhejiang University of Technology

Xiaolong Chen ( $\square$ richard_chen@zjut.edu.cn)

Institute of Fermentation Engineering https://orcid.org/0000-0003-3869-352X

\section{Research Article}

Keywords: a-glucosidase, transglycosylation, ethyl vanillin, rational design, site-direct mutation

Posted Date: October 26th, 2021

DOI: https://doi.org/10.21203/rs.3.rs-967592/v1

License: (c) (1) This work is licensed under a Creative Commons Attribution 4.0 International License.

Read Full License

Version of Record: A version of this preprint was published at Applied Biochemistry and Biotechnology on March 26th, 2022. See the published version at https://doi.org/10.1007/s12010-022-03908-6. 


\section{Abstract}

The $a$-glucosidase (EC 3.2.1.20) Agl2 produced by Xanthomonas campestris shows high a-glucosyl transfer activity toward alcoholic and phenolic hydroxyl groups. Ethyl vanillin- $\alpha$-glucoside, a precursoraroma compound with improved water solubility and thermal stability, can be synthesized through the transglycosylation of ethyl vanillin by Agl2. However, its low ethyl vanillin- $\alpha$-glucoside yield and ability to hydrolyze ethyl vanillin- $\alpha$-glucoside limits for industrial applications. Rational design and site-directed mutagenesis were employed to generate three variants of $X$. campestris a-glucosidase: L145I, S272T and L145I/S272T, which displayed improved transglycosylation activity toward EV The ethyl vanillin- $\alpha$ glucoside yield of L145I/S272T is the highest and is up to yield $52.41 \%$. Besides, L145I/S272T also remarkably diminished the hydrolysis activity toward the transglycosylation product EVG compared to Agl2. Our rational design based the catalytic mechanism of the $a$-glucosidase reaction proved to be effective for producing mutants with improved the ratio of transglycosylation/hydrolysis of $a$ glucosidase, which provides an important theoretical basis for further research on the reaction mechanism of $a$-glucosidase.

\section{Introduction}

Ethyl vanillin (3-ethoxy-4-hydroxybenzaldehyde, EV), the main phenolic component in vanilla products, is broadly used in food and pharmaceutical industry [1]. Nevertheless, the application of EV will be limited by volatility, low water solubility and low bioavailability. Glycosylation can dramatically improve the solubility and bioavailability of hydroxyl-containing small molecules, besides EV glycoside can be used as potential aromatic compounds [2, 3]. Ethyl vanillin- $\alpha$-glucoside (EVG) was firstly reported to be synthesized by environment-friendly one-step enzymatic transformation with a conversion rate of $33 \%$ [4]. The glycosylation efficiency of EV by biotransformation is still in the low level in comparison to that of structurally-related compound $/$-menthol [5].

Several types of carbohydrate-active enzymes (CAZymes) may be used in glycosylation reactions. Glycosyl transferases (GTs) require expensive nucleotide-activated sugars (e.g., uridine diphosphate glucose) as glycosyl donors, which hampers their application in industry [6]. Glycoside hydrolases (GHs) would be superior enzymes for glycosylation due to their natural abundance, robustness, wide acceptor specificity, and particularly, they are able to catalyze transglycosylation reactions starting from simple carbohydrates like maltose or sucrose [7]. Retaining GHs are the most studied for synthetic purpose, because they can transfer the glucose moiety of glycosyl donor to diverse acceptors through a double displacement mechanism (Fig. 1), without changing the configuration of the glycosidic bond [8]. Regrettably, the use of retaining GH for glycosylation of EV is weakened by their naturally hydrolytic activity, on account of water and EV (or glycosyl acceptor) are in a competitive relationship during deglycosylation [9]. Moreover, in hydrolytic retaining $\mathrm{GH}$, yield of EVG is further diminished by secondary hydrolysis, a reaction in which the synthetic product becomes a substrate for the enzyme. $a$-Anomerselective synthesis of EVG catalyzed by a GH 13-family a-glucosidase (Agl2) from Xanthomonas campestris was restrained both thermodynamically and kinetically [10]. Such low transglycosylation 
activity of Agl2 cannot be compensated by increasing the EV concentration to enhance affinity of Agl2 for EV, owing to the low solubility of EV [11]. Although increased transglycosylation/hydrolysis partition have been reported by addition of organic solvents [12], the enzyme in the reaction system never reached saturation and efficiency remained too low.

According to the double displacement mechanism, to increase the yield of glycoside products catalyzed by $a$-glucosidase, the affinity for glycosyl receptor in the deglycosylation process must be enhanced. For instance, modifying the residues in aglycone $(+)$ subsites around the catalytic pocket is a common strategy $[13,14]$. Johansson et al. reported that the aromatic amino acids in the +1 subsite are important for the interaction of GH 16-family enzymes (Xyloglucan endotransglycosylases) with glycosides [15]. In addition, higher transglycosylation activity of Neisseria polysaccharea amylosucrase to allyl 2acetamido-2-deoxy-a-D-glucopyranoside was also obtained by change the aglycone (+) subsites [16]. Equally, Hui et al. postulated that since deglycosylation is rate-limiting, an increase in receptor affinity would improve transglycosylation [17], higher transglycosylation ratios were also found in other mutational studies with increased affinity for +1 subsites [18]. An alternative strategy to enhance transglycosylation/hydrolysis $(\mathrm{T} / \mathrm{H})$ ratio involves modifying the residues around glycone (-) subsites to reduce the affinity for the glycosyl donor [19]. For example, substitution of several highly conserved amino acid residues near the -1 subsite of the Thermophilic thermophilus $\beta$-glycosidase increased its transglycosylation ability [20]. These mutations reduced hydrogen bonding interactions between highly conserved amino acids around -1 subsite and the donor substrate 2-deoxy-2-fluoro-D-glucosyl, then destabilize the transition state of the reaction, thereby increasing the $\mathrm{T} / \mathrm{H}$ ratio.

The catalytic domain of Agl2 contains typical $(\beta / \alpha)_{8}$ structural architecture. Employing maltose as glycosyl donor, it displays high transglycosylation activity toward alcoholic and phenolic hydroxyl groups without producing oligosaccharides as by-products [21]. Agl2 also contains an unique structure like $\beta \rightarrow a$ loop 4, which might be related to the diversity in glycosyl acceptor substrates [22]. In this study, we performed a structural-function analysis of Agl2 based on homology modelling and site-directed mutagenesis analysis, which directed to rationally design of Agl2 variants for improved EVG yields. Two strategies were adopted. The first strategy was to destroy the hydrogen bond between the Agl2 and maltose (glycosyl donor) by changing a conserved residue at -1 subsite. The second method was to increase the hydrophobicity around +1 subsite, which enhanced the affinity of Agl2 and EV (glycosyl acceptor).

\section{Materials And Methods}

\section{Strains and culture conditions}

The bacterial strains used in this study included Xanthomonas campestris sp. campestris 8004 and Escherichia coli BL21 [F-, ompT, hsdSB ( $\mathrm{rB}-, \mathrm{mB}-)$, dcm, gal, (DE3)]. The strain 8004 was cultivated in the NYG media at $30^{\circ} \mathrm{C}, 150 \mathrm{rpm}$ for $36 \mathrm{~h}$ [23], and E. coli BL21 (DE3) was cultivated in the LB (Luria-Bertani) medium at $37^{\circ} \mathrm{C}, 200 \mathrm{rpm}$. 


\section{Construction of mutants, expression, and purification of wild-type and mutant Agl2}

The genomic DNA of $X$. campestris was extracted using Bacterial DNA Extraction Kit (Omega Bio-tek, Guangzhou, China). The target gene encoding $a$-glucosidase (NCBI-Protein ID: AAY48708, Agl2) was amplified by high-fidelity PCR using The Phanta ${ }^{\circledR}$ Max Super-Fidelity DNA Polymerase Kit (Vazyme Biotech, Nanjing, China) and was purified by Gel Extraction Kit (Omega Bio-tek, Guangzhou, China). The plasmid pET28a was extracted using Plasmid Mini Kit I (Omega Bio-tek, Guangzhou, China), then was digested by restriction endonuclease Nde I and Hind III (TaKaRa Bio, Dalian, China) for $4 \mathrm{~h}$, then linearized pET28a were purified with Gel Extraction Kit. The purified Agl2 gene was cloned into the purified linearized pET28a between Nde I and Hind III sites by One Step Cloning Kit (Vazyme Biotech, Nanjing, China). The resulting plasmid, pET28a-Agl2, was used to transform E. coli, selecting for its presence in cells by the inclusion of $50 \mu \mathrm{g} \cdot \mathrm{mL}^{-1}$ kanamycin (Sangon Biotech, Shanghai, China) in the LB medium.

The site-directed mutagenesis was carried out using the Mut Express II Fast Mutagenesis Kit V2 (Vazyme Biotech, Nanjing, China). The DNA template for mutagenesis was the plasmid pET28a-Agl2 and mutations were introduced using pairs of complementary oligonucleotide primers (Table 1), designed at positions considered to be related to transglycosylation activity. Mutations were verified by DNA sequencing (Sangon Biotech, Shanghai, China) in both forward and reverse directions. Recombinant wildtype (WT) and mutant Agl2 were purified from E. coli BL21 (DE3) as previously described [10]. The protein concentrations of WT and mutants were determined using Modified Bradford Protein Assay Kit (Sangon Biotech, Shanghai, China).

\section{Determination of kinetic parameters}

The kinetic parameters of transglycosylation activity were investigated by purified Agl2 and its mutants. Various substrate concentrations of EV from $0.5 \mathrm{~g} / \mathrm{L}, 1.0 \mathrm{~g} / \mathrm{L}, 1.5 \mathrm{~g} / \mathrm{L}, 2.0 \mathrm{~g} / \mathrm{L}, 2.5 \mathrm{~g} / \mathrm{L}, 3.0 \mathrm{~g} / \mathrm{L}, 3.5 \mathrm{~g} / \mathrm{L}$ to $3.96 \mathrm{~g} / \mathrm{L}$ (maximum solubility of water phase system) were used in combination with $20 \mu \mathrm{L}$ of purified Agl2 or its mutant, $340 \mathrm{~g} / \mathrm{L}$ of maltose in $50 \mathrm{mM}$ of phosphate buffer solutions $(\mathrm{pH} 7.0)$. The initial rates were detected in the first 10 min according to the amount of EVG by adding $100 \mu \mathrm{L}$ reaction sample to $900 \mu \mathrm{L} 0.1 \mathrm{M} \mathrm{HCl}$ solution to quench the reaction. Experiments were performed in triplicate and the data of initial velocity were fitted with Michaelis-Menten equation to obtain the kinetic parameters $\left(k_{\text {cat }}\right.$ and $\left.V_{\max }\right)$.

\section{Quantitative analysis by HPLC}

HPLC (Agilent Technologies 1260 Infinity II, USA) with a DAD detector (Agilent Technologies G7115A 1260 DAD WR, USA) and an InertSustain C18 (GL Sciences, Japan) column was employed to quantify the concentrations of EV and EVG in the samples. The HPLC conditions were as follows: mobile phase, methanol-water (55:45, v/v); flow rate, $1 \mathrm{~mL} / \mathrm{min}$; temperature, $40^{\circ} \mathrm{C}$; injection volume, $10 \mu \mathrm{L}$; and wavelength, $272 \mathrm{~nm}$.

\section{Molecular dynamic simulation}


The coordinates from the crystal structure of Agl2 (PDB code 6AAV) were used as a starting point for the simulation. Mutations L145I, S272T and L145I/S272T with EV molecule were introduced by Scwrl4. GROMACS 5.0 was employed to perform the molecular dynamics (MD) simulation. OPLS-AA force field was used in the MD simulation, and the simulated target temperature was $318 \mathrm{~K}\left(45^{\circ} \mathrm{C}\right)$ and with an ionization state corresponding to $\mathrm{pH}$ 6.5. Agl2 and its mutants were placed in the center of a cube filled with 21,750 water molecules from a simple point-charged water model. Before MD simulation, the energy of the system was minimized and the system reached equilibrium through constant volume and temperature, then constant pressure and temperature. Simulations of 50 ns and 500 ns were performed with a time step of $2 \mathrm{fs}$, and the coordinates were saved every 5000 steps (10 ps).

\section{Influence of $\mathrm{pH}$ and temperature on transglycosylation activity}

The optimum $\mathrm{pH}$, optimum temperature, $\mathrm{pH}$ stability, and thermostability of wide-type enzyme and its mutants for transglycosylation activity were evaluated. The enzymes (Agl2 and its mutants) were used at the following concentrations: $0.81 \mathrm{~g} / \mathrm{L}$ for Agl2, $0.52 \mathrm{~g} / \mathrm{L}$ for $\mathrm{L} 145 \mathrm{l}, 0.66 \mathrm{~g} / \mathrm{L}$ for S272T, and $0.53 \mathrm{~g} / \mathrm{L}$ for L145I/S272T. One unit of glucoside transfer activity was defined as the amount of enzyme that produced $1 \mu \mathrm{mol}$ of EVG per minute in the given conditions.

The optimum $\mathrm{pH}$ for transglycosylation activity of the Agl2 and its mutants was evaluated in the standard reaction condition (each $1 \mathrm{~mL}$ of reaction mixture containing $3.96 \mathrm{~g} / \mathrm{L} \mathrm{EV}, 340 \mathrm{~g} / \mathrm{L}$ maltose and purified enzyme was incubated for $10 \mathrm{~min}$ at $40^{\circ} \mathrm{C}$ ), and $50 \mathrm{mM}$ different $\mathrm{pH}$ buffer (sodium acetate buffer for $\mathrm{pH}$ 4.0-5.5, PBS buffer for $\mathrm{pH}$ 6.0-8.0 and BSP buffer for $\mathrm{pH}$ 8.0-9.0).

Briefly, the optimum temperature was determined by assaying the transglycosylation activity from 30 to $50^{\circ} \mathrm{C}$ at its optimum $\mathrm{pH}$, respectively. Then the thermostability of $\mathrm{Agl} 2$ and its mutants were determined at optimum temperature. The solutions of Agl2 and its mutants were incubated in thermostatic water baths with different time ranging from 0 to $5 \mathrm{~h}$, then $20 \mu \mathrm{L}$ of Agl2 and its mutants' solutions were added in standard reaction condition for enzyme activity asay, respectively.

\section{Results And Discussion}

\section{Selection of mutation sites and mutant construction}

To determine the amino acid residues around the aglycone $(-1$ and +1$)$ subsites of Agl2, the threedimensional structure model of Agl2 was superimposed on that of HaG, one structurally-related $\mathrm{GH}$ 13family $a$-glucosidase. Its crystal structure contains the substrate maltose, which help to identify the key residues interacting with maltose [21]. Supplementary Fig. S1 showed a comparison of the amino acid residues for the maltose binding between Agl2 and HaG. Five amino acids H104, R199, E270, H330 and R398 were identified at the aglycone (-1) subsites of Agl2 as shown in Fig. 2. These amino acids were also conserved among GH 13-family glucosidases (Fig. 3), thereby they should play a role in binding the glycosyl donor. 4 GH 13-type glucosidases. As shown in Fig. 3, the multiple sequence alignment between 
Agl2 and 4 GH 13-family glucosidases like HaG, SmDG, GSJ and Pmg, E270 and H330 are completely conserved among the five glucosidases, H104, R199 and R398 are also conserved except for Pmg. Based on above structurally-related information, the catalytic functions of these five amino acid residues located at -1 subsite were predicted as showed in Table 2, which mainly formed a hydrogen bond network with the glycosyl donor maltose. Weakening the binding of glycosyl donor in -1 subsite by mutation of these residues might be effective for improving the $T / H$ ratio of glucosidases $[19,20,24]$. Therefore, these highly conserved amino acids around the -1 subsite (H104, R199, H330 and R398) was selected and replaced with neutral amino acid residues by site-mutagenesis method.

EV transglycosylation occurs when the EV molecule binds to the +1 subsite of Agl2 as an acceptor substrate. Increasing the affinity of +1 subsite for glycosyl acceptor EV can enhance its transglycosylation activity[16, 18]. The 4 amino acid residues, L145, A202, G227, and S272, are located at the +1 subsite of Agl2 according to the docking analysis (Supplementary Fig. S1). Based on multiple sequence alignment (Fig. 3), these residues are not conserved, replacing them with hydrophobic amino acids (especially aromatic residues) may increase the affinity for hydrophobic EV [13].

Taken together, the predicted relationships of structure and function for Agl2 were summarized in Table2. Based on this information, we designed mutants at position H104, R199, H330 and R398 to weaken the hydrogen bond interactions in - 1 subsite, and chose hydrophobic amino acids with various sizes (such as Ala, lle and Phe) for replacing each residue (L145, A202, E227, and S272) in +1 subsite.

\section{Influence of the mutations on the enzyme activity and the synthesis yield of EVG}

Changing in conserved residues at the -1 subsite can increase the transglycosylation/hydrolysis ratio [20], while this situation did not happen for EV glucosylation by Agl2. Replacement of the basic amino acids (H104, H330, R199, R398) forming putative hydrogen bonds with maltose near the -1 subsite with neutral amino acids (Ala and Cys) resulted in a dramatic decrease in EVG synthesis activity. Among these mutants, the relative activity of R398C, which had the highest transglycosylation activity toward EV, which only kept $45 \%$ of wild-type Agl2 (supplementary Fig. S3c). Moreover, the substitution with different basic amino acids also strongly impaired transglycosylation activity of Agl2. For example, mutants H104R, $\mathrm{H} 104 \mathrm{~K}, \mathrm{R} 199 \mathrm{~K}$ and R398K completely lost their activity, and R199H and R398H retained less than $20 \%$ of transglycosylation activity compared to Agl2. These results indicated that the presence of the hydrogen bond interactions between H104, R199, H330, R398 with maltose were crucial for catalytic capacity (Fig. 2).

It has been reported that the aromatic amino acid residues near the active sites of GHs commonly interact with the sugar ring, thereby affecting the transglycosylation reaction [14]. In order to increase the affinity for $E V$, the mutants at +1 subsite were constructed including L145F/W/Y, A202F/W/Y, G227F/W/Y and S272F/W/Y. However, all these mutants completely lost their transglycosylation activity towards EV. These results indicate that bulky aromatic amino acid residues should cause serious steric hindrance for EV molecule. 
In other hand, high hydrophobic but smaller residues such as isoleucine, valine, leucine were used for replacing these residues near the +1 subsites like L145, A202, G227 and S272. Among these mutants, only L145I and S272T displayed the improved transglycosylation activity. The synthetic capacity of EVG

and the kinetic parameters $\left(K_{\mathrm{m}}, k_{\mathrm{cat}}\right.$, and $\left.k_{\mathrm{cat}} / K_{\mathrm{m}}\right)$ of mutants were were compared with the wild-type Agl2 (Table 3). $K_{\mathrm{m}}$ value of L145I (10.22 mM) was lower than that of Agl2 $(27.12 \mathrm{mM})$, which showed its affinity for EV increased. Its $k_{\text {cat }} / K_{\mathrm{m}}$ value was 5.76 times than that of Agl2. For the mutants at residue S272, according to supplementary table S1, S272I, S272L and S272V displayed an increased affinity for EV (their $K_{\mathrm{m}}$ values were 15.41, 20.71 and 25.14 , respectively), but did not increase transglycosylation activity compared to Agl2. For mutant S272T, $K_{\mathrm{m}}$ value $(28.04 \mathrm{mM})$ was similar to the wild-type enzyme, but its $k_{\text {cat }} / K_{\mathrm{m}}$ value was enhanced. Therefore, the double mutant L145I/S272T was constructed, which had the $K_{m}$ value of $6.17 \mathrm{mM}$, meaning highest affinity for EV. Besides, it had the highest efficiency in catalyzing the production of EVG among all the mutants according to its $k_{\text {cat }} / K_{\mathrm{m}}$ value was 4.69 $\mathrm{mM}^{-1} \cdot \mathrm{s}^{-1}$.

\section{Molecular mechanisms associated with increased EVG yield}

The mutant L145I/S272T generated the highest EVG yield due to the synergistic effects of these two sites, as shown in Fig. 3, L145 of Agl2 is not conserved in the GH 13-family. The position of L145 is involved in glycosyl acceptor binding site ( +1 subsite) and multiple mutants in this position displayed higher affinity for EV. Among these mutants, L145I displayed the highest EVG production (Supplementary Fig. S3). This result indicates that the replacement by the more hydrophobic, but similar size isoleucine at position L145 effectively promote the transglycosylation ability of Agl2.

The residue $\mathrm{S} 272$ is located at the $\beta$-sheet of the main catalytic domain A of Agl2, which is separated from catalytic acid/base E270 by one amino acid. S272 is not conserved among the enzymes of GH 13family (Fig. 3) and is important for glycosyl acceptor specificity. It is reported that substituting S272 with an aliphatic amino acid led to the enhancement of $\alpha$-glucosylation activity of Agl2 toward hydroquinone [21]. In contrast, among various mutants at position 272, only S272T showed increased transglycosylation activity toward EV compared to the wild-type Agl2 (Supplementary Fig. S3).

Replacement by threonine at this position means few changes in hydrophobicity and steric hindrance, but might produce the synergistic conformational effect on the orientation and dynamic property of adjacent catalytic residue E270. According to Reetz et al, two amino acids that are spatially close to each other exist a potential synergistic conformational effected by the orientation of their side chains [25]. As for Agl2, its catalytic acid/base (E270) is located at position 270, and it is speculated that the serine at position 272 may have a potential synergistic conformational effect with E270 based on its position on the $\beta$-sheet. Therefore, we speculate that the change of the amino acid residue at position 272 affects the orientation of catalytic acid/base, thereby affecting the hydrolysis/transglycosylation reaction, subsequent MD simulations also confirmed this speculation. 
MD simulation was applied to explore the molecular-level changes of mutants compared to wild-type Agl2. The distortion of catalyzed acid/base for mutant S272T and L145I/S272T were observed during 50 ns and 500 ns MD simulation analysis, while no such distortion was seen for wild-type Agl2 (Fig. 4). Meanwhile, the distance from the oxygen ion of the catalytic base (E270) of S272T and L145I/S272T to the hydrogen atom on hydroxyl group of the EV was increased nearly 2-fold compared to wild-type Agl2 (Fig. 5). This phenomenon can be explained by different hypotheses. First, incorrect positioning of the catalytic base would prevent EV/water from binding to glycosyl-enzyme intermediate and thereby extend the lifetime of the glycosyl-enzyme intermediate [26]. Increasing the lifetime of the glycosyl-enzyme intermediate permits more time for EV to enter the active site. On the other hand, as described by Kelly et al for a type of GH 13-family CGTase [27], the catalytic conformation could be restored by glycosyl acceptor (EV) binding, thus the enzyme cannot use water as acceptor. Another explanation is related to catalytic water, the only non-aqueous mediated interaction with potentially catalytic water in the crystal structure of TnBgl1A is acid/base. Therefore, twisting acid/base would lock the catalytic water in a position that is not conducive to hydrolysis, similar to the proposed natural TG mechanism [28]. In summary, the distortion of the catalytic acid/base E270 was observed for the mutants S272T and L145I/S272T, which demonstrated E270 was not only a catalytic acid/base but also vital for directing the catalytic water.

\section{Optimization of EVG production}

The optimum pH of mutant L145I, S272T and L145I/S272T were all at pH6.5, the same with that of Agl2 (Fig. 6a). The initial transglycosylation activity of wild-type and its mutants both gradually increased in the area of $\mathrm{pH} 4.0-6.5$, but their activity dropped rapidly as the $\mathrm{pH}$ of the reaction environment exceeds 6.5. We further optimized the $\mathrm{pH}$ between 5.8-7.0 to detect initial transglycosylation activity of Agl2 and its mutants. As shown in Fig. 6b, all 4 enzymes reach the maximum transglycosylation activity at the pH6.5, which illustrated that the pH properties of mutants L145I, S272T and L145I/S272T had not altered for catalyzing EV to EVG.

The optimal temperatures and thermal stability of L145I, S272T and L145I/S272T were compared to those of WT. The optimal temperatures of the mutants and WT were almost indistinguishable, as the temperature increased, the EVG concentration reached a peak at $45^{\circ} \mathrm{C}$ and then decreased (Fig. $7 \mathrm{a}$ ). However, as shown in Fig. 7b, the initial enzyme activity of Agl2 and its mutants increased with the increase of temperature ranging from 30 to $55^{\circ} \mathrm{C}$. It was confirmed that, when the reaction temperature exceeds $45^{\circ} \mathrm{C}$, the enzyme was inactivated faster than it was activated. Therefore, the optimal temperature for catalyzing EVG is $45^{\circ} \mathrm{C}$. In addition, it could be seen from Fig. 7c that the thermal stability of the mutants had no significant change compared to the thermal stability of Agl2.

The maximum solubility of $\mathrm{EV}$ in water at $4^{\circ} \mathrm{C}$ is $3.96 \mathrm{~g} / \mathrm{L}$. When the maximum concentration of EV was used in the reaction catalyzed by $\mathrm{Agl} 2$, the maximum conversion rate was $40.98 \%$, and the concentration of EVG could reach $3.25 \mathrm{~g} / \mathrm{L}$. As mentioned above, the enzyme reactions were carried out under optimal conditions $\left(\mathrm{pH} 6.5,45^{\circ} \mathrm{C}\right)$ in order to investigate the changes in EVG production over time. The 
corresponding EVG concentrations of the AgI2 and L145I/S272T were monitored during the glycosylation process (Fig. 8). During the first $1 \mathrm{~h}$, EVG yield increased rapidly until it reached the maximum.

Subsequently, degradation of the EVG was observed with prolonged reaction time instead of reaching equilibrium. The EVG yield for Agl2 reached the maximum $(41.1 \%, 3.25 \mathrm{~g} / \mathrm{L})$ at $0.5 \mathrm{~h}$, and that for $\mathrm{L} 145 \mathrm{I} / \mathrm{S} 272 \mathrm{~T}$ reached the maximum $(52.8 \%, 4.17 \mathrm{~g} / \mathrm{L})$ at $0.75 \mathrm{~h}$. The maximum yield of L145I/S272T was increased by $11.7 \%$ relative to Agl 2 .

\section{Conclusion}

In this study, two single mutants (L145I and S272T) and one double mutant (L145I/S272T) of Agl2 were constructed, and their EVG yield from EV was determined. L145I, S272T and L145I/S272T all produced EVG yield higher than that of Agl2, in other words, these mutants had lower hydrolysis activity toward EVG compared with Agl2. L145I/S272T produced the highest EVG yield (52.8\%) reported so far, which solved the bottleneck that the transglycosylation product EVG would be massively hydrolyzed by Agl2, and significantly improved its potential industrial applications.

L145I increased +1 subsite hydrophobicity to enhance transglycosylation. MD simulation demonstrated that the E270 of S272T or L145I/S272T was distorted, which is not only a catalytic residue, but also important for reducing the hydrolysis activity of EVG. The activity of L145I/S272T reflects an additive effect of the two single mutants to further improve the transglycosylation activity of Agl2. This study supplied an efficient method engineer the transglycosylation activities of an enzyme from GH 13-family, which provides an important theoretical basis for further research on the reaction mechanism of $a$ glucosidase.

\section{Declarations}

Funding The financial support provided for this project was from the National Natural Science Foundation of China (Grant No. 21908196).

Ethical Approval Not applicable.

Data Availability All data sources could be available to readers on request.

Code Availability Not applicable.

Consent to Participate Not applicable.

Consent to Publish Not applicable.

Conflict of Interest The authors declare no competing interests.

\section{Author Contribution}


LY Chen: Conceptualization, methodology, validation, formal analysis, investigation \& writing original draft.

Y Liu: Investigation.

YY Zhou: Review \& editing

LJ Zhu \& XL Chen: Conceptualization, funding acquisition, supervision, writing, review \& editing.

\section{References}

1. Jung, H. J., Song, Y. S., Kim, K., Lim, C. J., \& Park, E. H. (2010) Assessment of the anti-angiogenic, antiinflammatory and antinociceptive properties of ethyl Vanillin. Archives of Pharmacal Research, 33(2), 309-316.

2. Desmet, T., Soetaert, W., Bojarova, P., Kren, V., Dijkhuizen, L., Eastwick-Field, V., \& Schiller, A. (2012) Enzymatic glycosylation of small molecules: challenging substrates require tailored catalysts. Chemistry A European Journal 18(35), 10786-10801.

3. Desmet, T., \& Soetaert, W. (2011) Enzymatic glycosyl transfer: mechanisms and applications. Biocatalysis and Biotransformation, 29(1), 1-18.

4. Kim, S. K., Kim, K. S., Ra, D. Y., \& Kim, Y. H. (2003) Enzymatic synthesis of vanillin- $\alpha$-glucoside and ethyl vanillin-a-glucoside. Journal of the Koresn Society of Tobacco Science, 25(2), 120-127.

5. Chen, L. Y., Zhou, Y. L., Lu, C. X., Ma, Z., Chen, H. C., Zhu, L. J., Lu, Y. L., \& Chen, X. L. (2021) Efficient production of $/$-menthyl $\alpha$-glucopyranoside from $/$-menthol via whole-cell biotransformation using recombinant Escherichia coli. Biotechnology Letters 43(2), 1757-1764.

6. Bungaruang, L., Gutmann, A., \& Nidetzky, B. (2013) Leloir glycosyltransferases and natural product glycosylation: biocatalytic synthesis of the C-glucoside nothofagin, a major antioxidant of redbush herbal tea. Advanced Synthesis \& Catalysis, 355(14-15), 2757-2763.

7. Okuyama, M., Saburi, W., Mori, H., \& Kimura, A. (2016) $a$-Glucosidases and $a-1,4$-glucan lyases: structures, functions, and physiological actions. Cellular and Molecular Life Sciences, 73(14), 2727-2751.

8. Koshland, D. E. (1953) Stereochemistry and the mechanism of enzymatic reactions. Biological reviews, 28(4), 416-436.

9. Crout, D., \& Vic, G. (1998) Chemlnform abstract: glycosidases and glycosyl transferases in glycoside and oligosaccharide synthesis. Chem/nform, 2(1), 98-111.

10. Chen, H. C., Yang, S. S., Xu, A. J., Jiang, R. N., Tang, Z. C., Wu, J. M., Zhu, L. J., Liu, S. J., Chen, X. L., \& Lu, Y. L. (2019) Insight into the glycosylation and hydrolysis kinetics of $a$-glucosidase in the synthesis of 
glycosides. Applied Microbiology and Biotechnology, 103(23-24), 9423-9432.

11. Banerjee, G., \& Chattopadhyay, P. (2019) Vanillin biotechnology: the perspectives and future. Journal of the Science of Food and Agriculture, 99(2), 499-506.

12. Winter, K., Desmet, T., Devlamynck, T., Renterghem, L., Verhaeghe, T., Pelantova, H., Kren, V., \& Soetaert, W. (2014) Biphasic catalysis with disaccharide phosphorylases: chemoenzymatic synthesis of $a$-DGlucosides using sucrose phosphorylase. Organic Process Research \& Development, 18(6), 781-787.

13. Armand, S., Andrews, S. R., Charnock, S. J., \& Gilbert, H. J. (2001) Influence of the aglycone region of the substrate binding cleft of Pseudomonas xylanase 10A on catalysis. Biochemistry, 40(25), 7404-7409.

14. Taira, T., Fujiwara, M., Dennhart, N., Hayashi, H., Onaga, S., Ohnuma, T., Letzel, T., Sakuda, S., \& Fukamizo, T. (2010) Transglycosylation reaction catalyzed by a class V chitinase from cycad, Cycas revoluta: a study involving site-directed mutagenesis, HPLC, and real-time ESI-MS. Biochimica et Biophysica Acta (BBA) - Proteins and Proteomics, 1804(4), 668-675.

15. Johansson, P., Brumer, H., Baumann, M. J., Kallas, A. M., Henriksson, H., Denman, S. E., Teeri, T. T., \& Jones, T. A. (2004) Crystal structures of a poplar xyloglucan endotransglycosylase reveal details of transglycosylation acceptor binding. Plant Cell, 16(4), 874-886.

16. Champion, E., Guerin, F., Moulis, C., Barbe, S., Thu Hoai, T., Morel, S., Descroix, K., Monsan, P., Mourey, L., Mulard, L. A., Tranier, S., Remaud, M., \& Andre, I. (2012) Applying pairwise combinations of amino acid mutations for sorting out highly efficient glucosylation tools for chemo-enzymatic synthesis of bacterial oligosaccharides. Journal of the American Chemical Society, 134(45), 18677-18688.

17. Hui, Y. F., Drone, J., Hoffmann, L., Tran, V., Tellier, C., Rabiller, C., \& Dion, M. (2005) Converting a $\beta$ glycosidase into a $\beta$-transglycosidase by directed evolution. Journal of Biological Chemistry, 280(44), 37088-37097.

18. Lu, Y., Yang, H. T., Hu, H. Y., Wang, Y., Rao, Z. H., \& Jin, C. (2009) Mutation of Trp137 to glutamate completely removes transglycosyl activity associated with the Aspergillus fumigatus AfChiB1. Glycoconjugate Journal, 26(5), 525-534.

19. Arab-Jaziri, F., Bissaro, B., Tellier, C., Dion, M., Fauré, R., \& O’Donohue, M. J. (2015) Enhancing the chemoenzymatic synthesis of arabinosylated xylo-oligosaccharides by GH51 a-tarabinofuranosidase. Carbohydrate Research, 401(12), 64-72.

20. Teze, D., Hendrickx, J., Czjzek, M., Ropartz, D., Sanejouand, Y. H., Tran, V., Tellier, C., \& Dion, M. (2014) Semi-rational approach for converting a GH1-glycosidase into a $\beta$-transglycosidase. Protein Engineering Design \& Selection Peds, 27(1), 13-19.

21. Watanabe, R., Arimura, Y., Ishii, Y., \& Kirimura, K. (2020) Crystal structure of $\alpha$-glucosyl transfer enzyme XgtA from Xanthomonas campestris WU-9701. Biochemical and Biophysical Research Communications, 
$526(3), 580-585$.

22. Shen, X., Saburi, W., Gai, Z., Kato, K., \& Yao, M. (2015) Structural analysis of the $a$-glucosidase HaG provides new insights into substrate specificity and catalytic mechanism. Acta Crystallographica Section D, 71(6), 1382-1391.

23. Turner, P., Barber, C., \& Daniels, M. (1984) Behaviour of the transposons Tn5 and Tn7 in Xanthomonas campestris pv. campestris. Molecular and General Genetics 195(1-2), 101-107.

24. Bissaro, B., Durand, J., Biarnes, X., Planas, A., Monsan, P., O'Donohue, M. J., \& Faure, R. (2015) Molecular design of non-leloir furanose-transferring enzymes from an $\alpha$-tarabinofuranosidase: a rationale for the engineering of evolved transglycosylases. Acs Catalysis, 5(8), 4598-4611.

25. Reetz, M. T., Bocola, M., Carballeira, J. D., Zha, D. X., \& Vogel, A. (2005) Expanding the range of substrate acceptance of enzymes: combinatorial active-site saturation test. Angewandte ChemieInternational Edition, 44(27), 4192-4196.

26. Lundemo, P., Karlsson, E. N., \& Adlercreutz, P. (2017) Eliminating hydrolytic activity without affecting the transglycosylation of a GH1 $\beta$-glucosidase. Applied Microbiology and Biotechnology, 101(3), 11211131.

27. Kelly, R. M., Leemhuis, H., Rozeboom, H. J., van Oosterwijk, N., Dijkstra, B. W., \& Dijkhuizen, L. (2008) Elimination of competing hydrolysis and coupling side reactions of a cyclodextrin glucanotransferase by directed evolution. Biochemical Journal, 413(3), 517-525.

28. Larsbrink, J., Izumi, A., Hemsworth, G. R., Davies, G. J., \& Brumer, H. (2012) Structural enzymology of cellvibrio japonicus Agd31B protein reveals $\alpha$-transglucosylase activity in glycoside hydrolase family 31. The Journal of Biological Chemistry,287(52), 43288-43299.

\section{Tables}

Table 1. Partial oligonucleotides used in this study 


\begin{tabular}{|c|c|}
\hline Primer name & Sequence $^{\mathrm{a}}\left(5^{\prime} \rightarrow 3^{\prime}\right)$ \\
\hline H104A-F & Gtgatgatcgatcaggtactgagcgcctcctcgatcgcgcatg \\
\hline H104C-F & Gtgatgatcgatcaggtactgagctgêtcctcgatcgcgcatg \\
\hline H104-R & Agtacctgatcgatcatcactttcaacccaaggccatgtgcc \\
\hline L145A-F & Ctggctgtcggcctttggtggggtcgcatggcagtgg \\
\hline L145A-R & Caccaaaggçcgacagccagttgttcggcggcgttc \\
\hline L145I-F & Ctggctgtcgatctttggtggggtcgcatggcagtgg \\
\hline L145I-R & Caccaaagatcgacagccagttgttcggcggcgttc \\
\hline L145F-F & Ctggctgtcgttctttggtggggtcgcatggcagtgg \\
\hline L145F-R & Caccaaagaacgacagccagttgttcggcggcgttc \\
\hline R199A-F & Atcgcggtgtggatgggttcg $\underline{c c c t g g a t g c g a t c a a c}$ \\
\hline R199C-F & Atcgcggtgtggatgggttctgçctggatgcgatcaac \\
\hline R199-R & Catccacaccgcgatcgagccagaaccgcacgttatc \\
\hline A202I-F & Ccgtctggatatcatcaacttctgctttcacgacgcgc \\
\hline A202I-R & Gttgatgatatccagacggaacccatccacaccgcg \\
\hline A202F-F & Ccgtctggattttatcaacttctgctttcacgacgcgc \\
\hline A202F-R & Gttgataaaatccagacggaacccatccacaccgcg \\
\hline G227-F & Gcgcggacaatccttacgcctaccagtatc \\
\hline G227A-R & Taaggattgtccgcgctgaatgcgcgccccac \\
\hline G227I-R & Taaggattgtccgcgctgaagatgcgccccac \\
\hline G227F-R & Taaggattgtccgcgctgaagaagcgccccac \\
\hline S272A-F & Cttggcgagatcgçgtcggaagattcgctggccaccac \\
\hline S272A-R & Cgaatcttccgaçgcgatctcgccaaggctcactgcattc \\
\hline S272T-F & Cttggcgagatcacgtcggaagattcgctggccaccac \\
\hline S272T-R & Cgaatcttccgacgtgatctcgccaaggctcactgcattc \\
\hline S272F-F & Cttggcgagatcttctcggaagattcgctggccaccac \\
\hline S272F-R & Cgaatcttccgagaagatctcgccaaggctcactgcattc \\
\hline H330A-F & Gccatgctgggccatttccaatgçggacgtagtgcgc \\
\hline H330C-F & Gccatgctgggecatttccaattgtgacgtagtgcgc \\
\hline
\end{tabular}

Page 13/19 


\begin{tabular}{|ll|}
\hline H330-R & Aatggcccagcatggccagccctccaacatggtggcc \\
\hline R398A-F & Ctggccgaccttcaagggcgçggatggctgccgtacg \\
\hline R398C-F & Ctggccgaccttcaagggctgçgatggctgccgtacg \\
\hline R398-R & Cttgaaggtcggccagaaagttattccatacggatcc \\
\hline
\end{tabular}

a The mutated bases are underlined

Table 2. Putative function of the eight amino acids near the substrate-recognition site.

\begin{tabular}{|c|c|c|}
\hline Residue & Potential function & Changes in transglycosylation activity of mutants \\
\hline $\begin{array}{l}\mathrm{H} 104(-1 \\
\text { subsite) }\end{array}$ & $\begin{array}{l}\text { Binding of glucosyl moiety }(\mathrm{H}- \\
\text { bond with } \mathrm{OH} \text { ) }\end{array}$ & Mutants completely lost their activity toward EV \\
\hline $\begin{array}{l}\text { R199 (-1 } \\
\text { subsite) }\end{array}$ & $\begin{array}{l}\text { Binding of glucosyl moiety } \\
\text { (H-bond with } \mathrm{OH} 2 \text { ) }\end{array}$ & $\begin{array}{l}\mathrm{R} 199 \mathrm{H} \text { retained } 18 \% \text { of the transglycosylation } \\
\text { activity compared to Agl2 }\end{array}$ \\
\hline $\begin{array}{l}\text { H330 (-1 } \\
\text { subsite) }\end{array}$ & $\begin{array}{l}\text { Binding of glucosyl moiety } \\
\text { (H-bond with } \mathrm{OH} 3 \text { ) }\end{array}$ & Mutants completely lost their activity toward EV \\
\hline $\begin{array}{l}\text { R398 }(-1 \\
\text { subsite })\end{array}$ & $\begin{array}{l}\text { Binding of glucosyl moiety } \\
\text { (H-bond with } \mathrm{OH} 4)\end{array}$ & $\begin{array}{l}\mathrm{R} 398 \mathrm{H} \text { retained } 15 \% \text { of the transglycosylation } \\
\text { activity compared to Agl2 }\end{array}$ \\
\hline $\begin{array}{l}\text { L145 (+1 } \\
\text { subsite) }\end{array}$ & Pi-alkyl interaction & $\begin{array}{l}\text { The transglycosylation activity of L145I toward EV } \\
\text { was } 121 \% \text { of Agl } 2\end{array}$ \\
\hline $\begin{array}{l}\text { A202 }(+1 \\
\text { subsite })\end{array}$ & Alkyl interaction & $\begin{array}{l}\text { No mutant had higher transglycosylation activity } \\
\text { toward EV than Agl2 }\end{array}$ \\
\hline $\begin{array}{l}\text { G227 (+1 } \\
\text { subsite) }\end{array}$ & $\begin{array}{l}\text { Carbon hydrogen bond } \\
\text { interaction }\end{array}$ & $\begin{array}{l}\text { No mutant had higher transglycosylation activity } \\
\text { toward EV than Agl2 }\end{array}$ \\
\hline $\begin{array}{l}\text { S272 (+1 } \\
\text { subsite) }\end{array}$ & $\begin{array}{l}\text { Carbon hydrogen bond } \\
\text { interaction }\end{array}$ & $\begin{array}{l}\text { The transglycosylation activity of L145I toward EV } \\
\text { was } 145 \% \text { of Agl } 2\end{array}$ \\
\hline
\end{tabular}

Table 3. Kinetic parameters of EV transglycosylation for Agl2 and its mutants

\begin{tabular}{|llll|}
\hline Enzyme & $K_{\mathrm{m}}(\mathrm{mM})$ & $k_{\mathrm{cat}}\left(\mathrm{s}^{-1}\right)$ & $k_{\text {cat }} / K_{\mathrm{m}}\left(\mathrm{mM}^{-1} \cdot \mathrm{s}^{-1}\right)$ \\
\hline Agl2 (WT) & 27.12 & 10.47 & 0.38 \\
\hline L145I & 10.22 & 22.41 & 2.19 \\
\hline S272T & 28.04 & 23.51 & 0.84 \\
\hline L145I/S272T & 6.17 & 29.01 & 4.69 \\
\hline
\end{tabular}



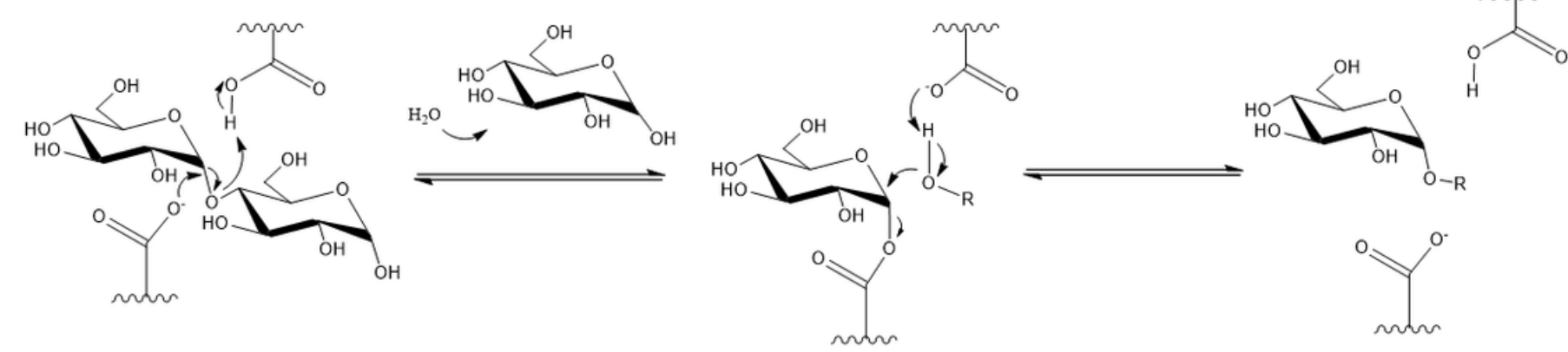

Figure 1

The double displacement mechanism of retaining a-glucosidases.

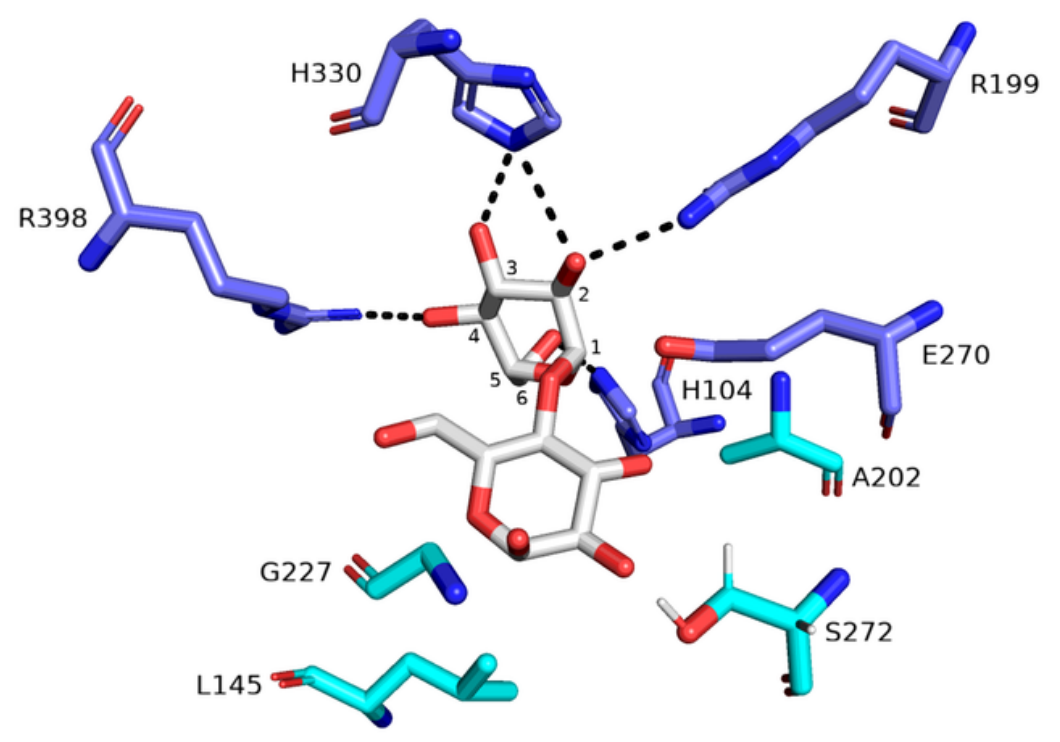

Figure 2

Close-up of active center region in Agl2 (PDB entry 6AAV), five amino acid residues around - 1 subsites were showed in navy blue stick, four amino acid residues around +1 subsites were showed in cyan stick. The white ligand was maltose, black dotted line represented the predicted hydrogen bond. 


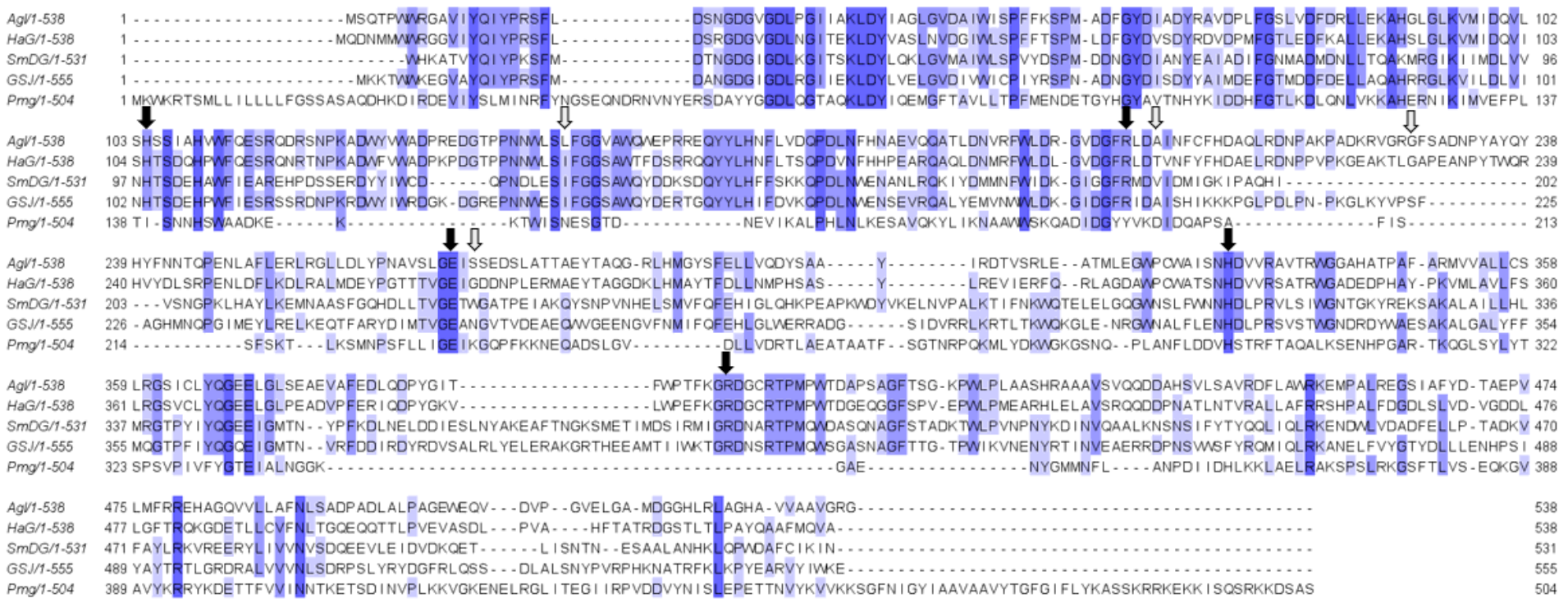

\section{Figure 3}

Sequence alignment of Agl2 and other a-glucosidases. Sequences were compared with the following enzyme/source/accession numbers: Agl2, Xanthomonas campestris pv. campestris str. 8004 (NCBIProtein ID: AAY48708); HaG, Halomonas sp. Strain H11 (NCBI-Protein ID: BAL49684); SmDG, Streptococcus mutans (NCBI-Protein ID: BAE79634); GSJ, Geobacillus sp. HTA-462 (PDB ID: 2ze0); Pmg, Priestia megaterium (GeneBank: WP_050691052.1). The alignment was generated with Clustal Omega. The regions of sequence identity were shaded in blue, and the regions of sequence similarity were in light blue. The amino acids selected in -1 subsite for mutagenesis were marked by black arrows in Agl2, the amino acids selected in +1 subsite for mutagenesis were marked by gray arrows.

a)

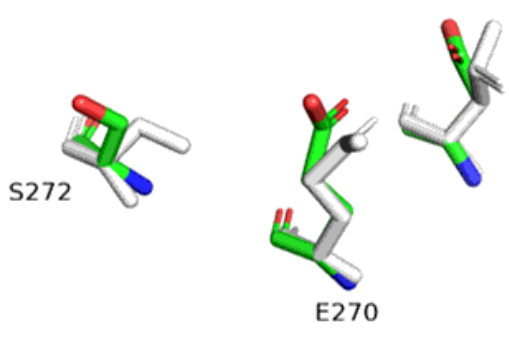

b)

D201

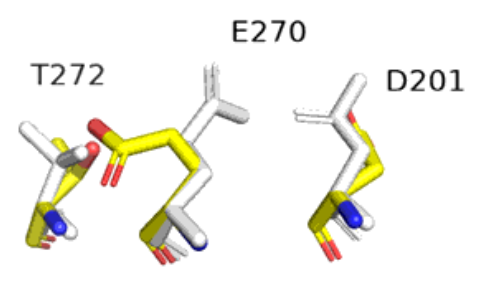

c)

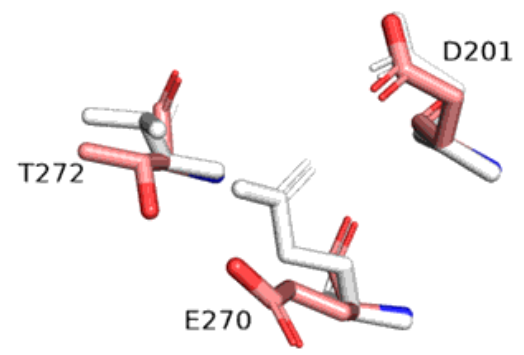

\section{Figure 4}

The superimposed structure of catalytic nucleophile, acid/base and residue at position S272 of Agl2, S272T and L145I/S272T at different time-points of a molecular dynamics simulation. (a) White: wild-type after 50 ns MD simulation, green: wild-type after 500 ns MD simulation; (b) white: S272T after 50 ns MD 
simulation, yellow: S272T after 500 ns MD simulation; (c) white: L145I/S272T after 50 ns MD simulation, orange: L145I/S272T after 500 ns MD simulation.

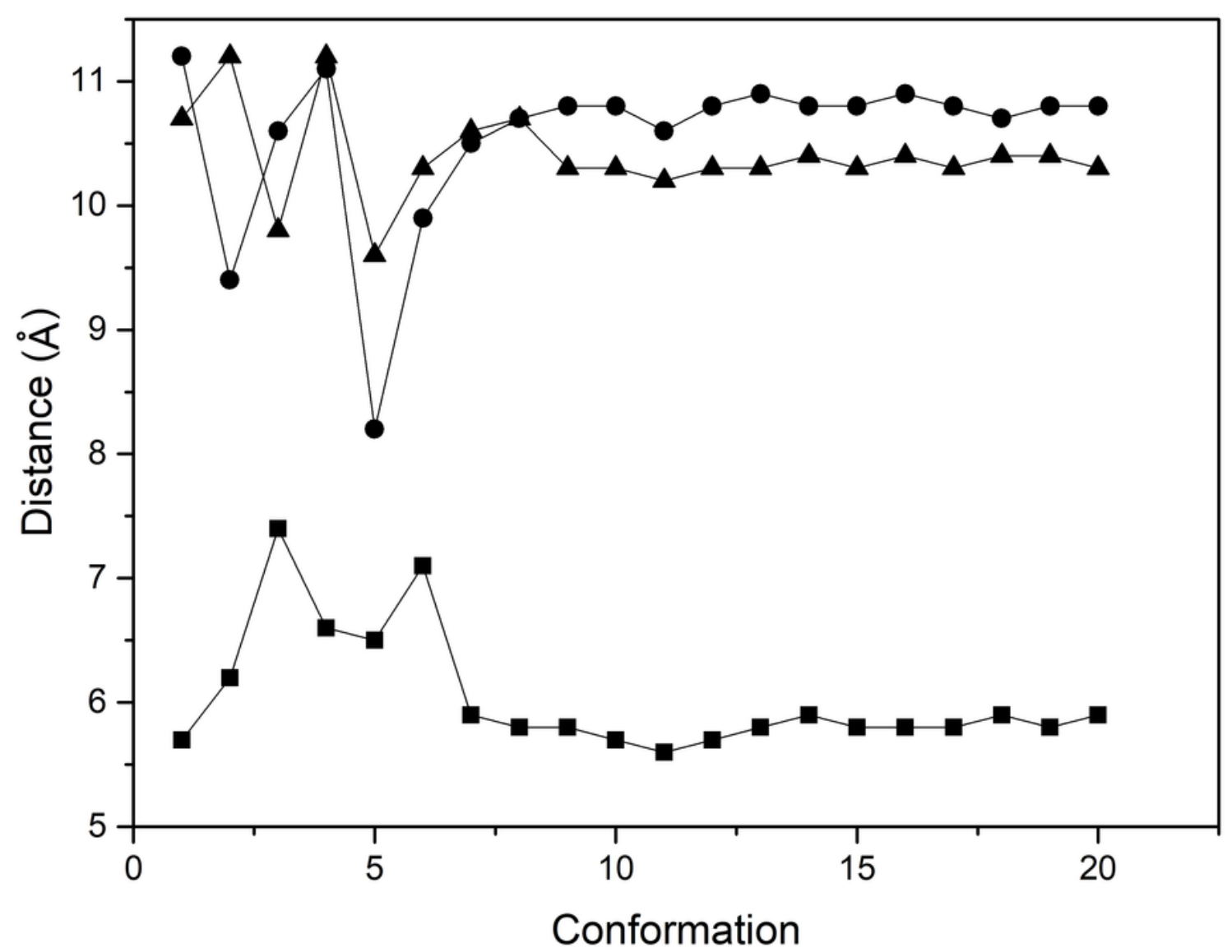

Figure 5

The distance from the oxygen ion of the catalytic base of Agl2 (Squares), S272T (circles) and L145I/S272T (up triangles) to the hydrogen atom on the hydroxyl group of the EV, the trajectory was analyzed by the results of MD simulation, 20 conformations were selected in each simulation. 

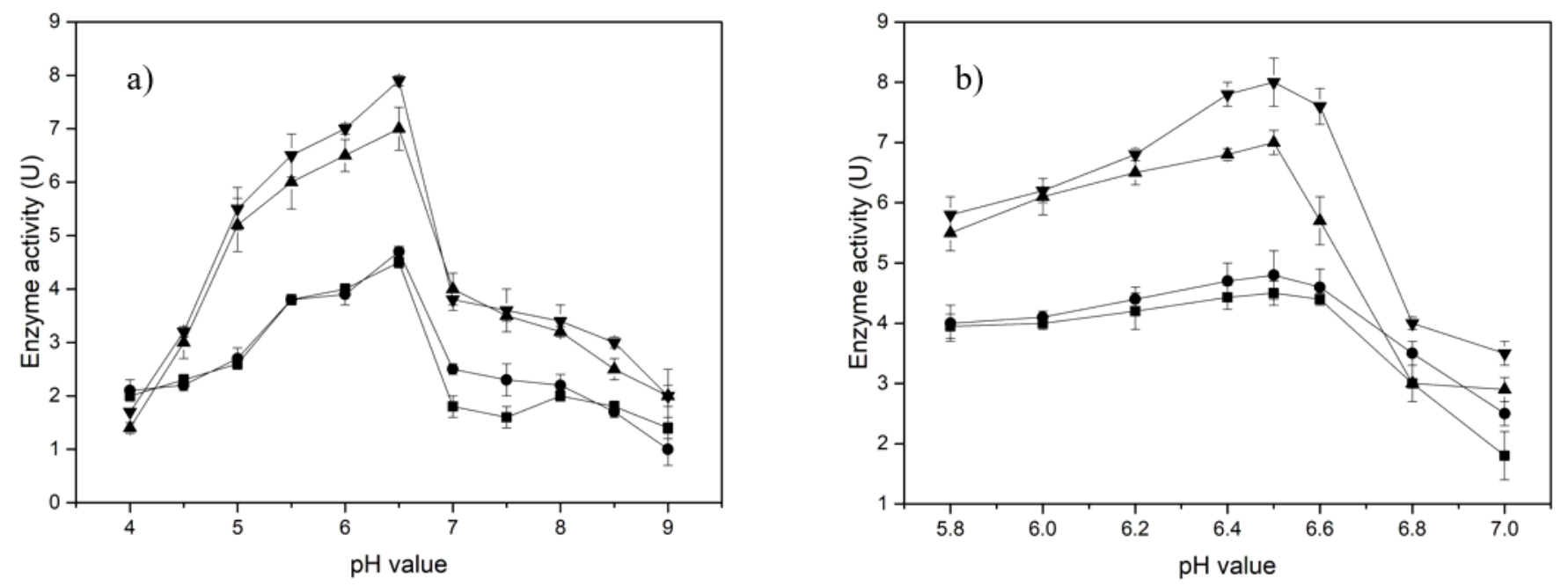

Figure 6

Effects of pH on transglycosylation activity of wide-type enzyme Agl2 (squares) and mutant L145I (up triangles), S272T (circles) and L145I/S272T (down triangles). (a) The optimum pH of Agl2, L145I, S272T and L145I/S272T were determined in buffers ranging from $\mathrm{pH} 4.0$ to 9.0. The condition of (b) was same as (a) except the buffers ranging from $\mathrm{pH}$ 5.8-7.0.
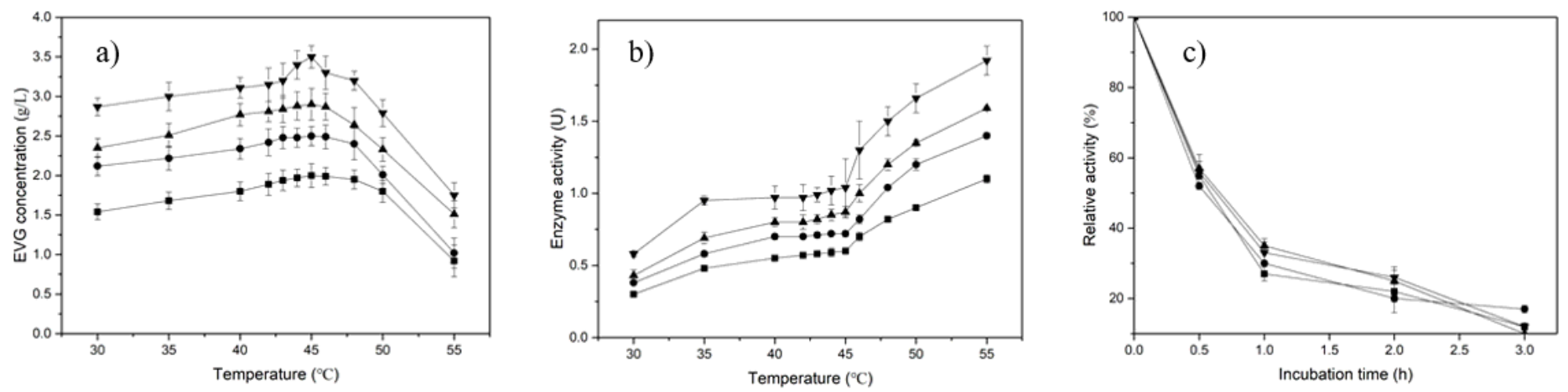

Figure 7

Effects of temperature on transglycosylation activity and thermal stability of wide-type enzyme Agl2 (squares) and mutant L145I (up triangles), S272T (circles) and L145I/S272T (down triangles). (a) The optimum temperatures for EVG synthesis of Agl2, L145I, S272T and L145I/S272T at temperatures ranging from 30 to $55^{\circ} \mathrm{C}$; (b) Initial transglycosylation activity of AgI2, L145I, S272T and L145I/S272T at temperatures ranging from 30 to $55^{\circ} \mathrm{C}$; (c) Relative activity of Agl2, L145I, S272T and L145I/S272T after incubation at $45^{\circ} \mathrm{C}$ for $0-5 \mathrm{~h}$. 


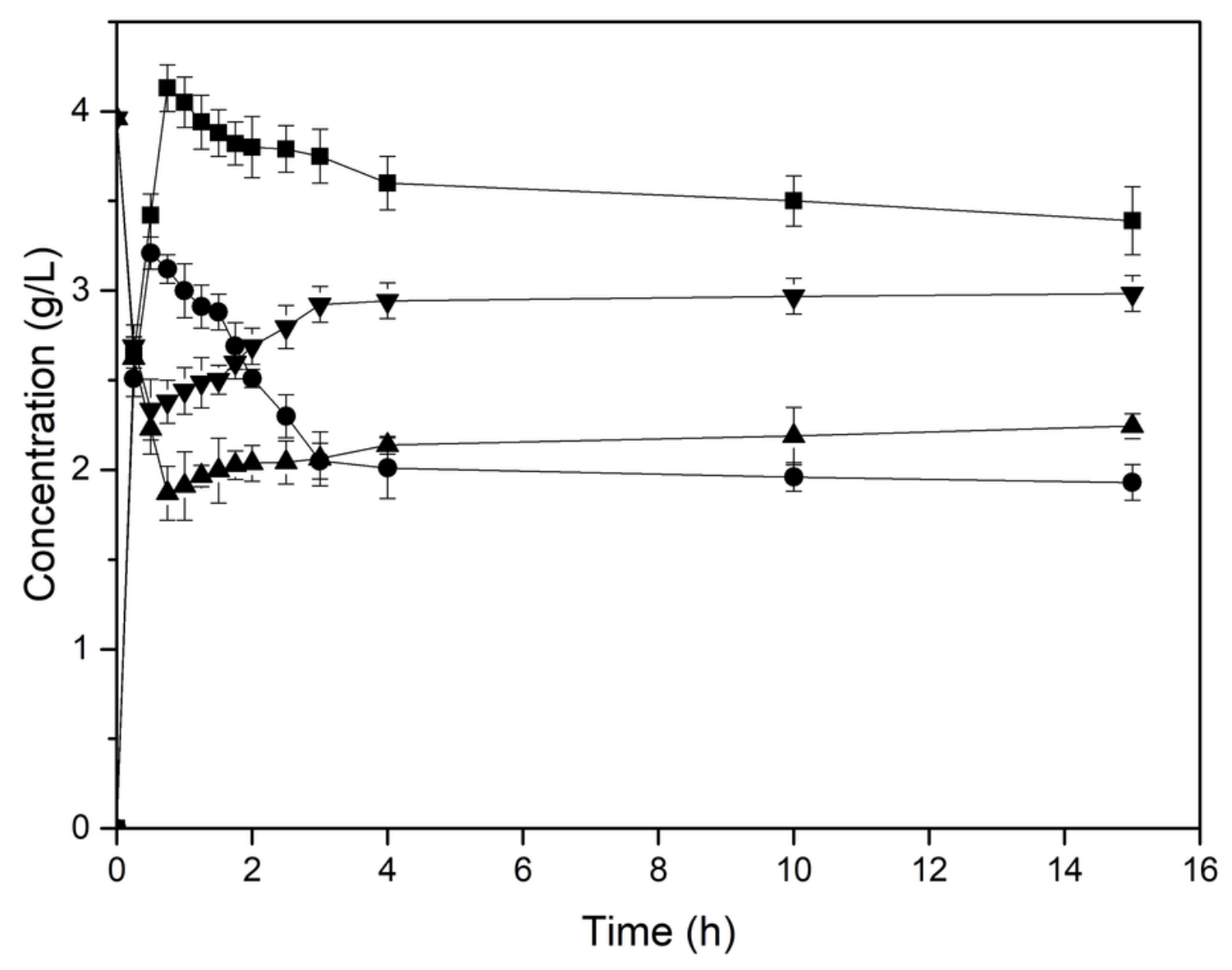

Figure 8

The glycosylation of EV catalyzed by Agl2 and L145I/S272T under optimal reaction conditions. The 980 $\mu \mathrm{L} 50 \mathrm{mM}$ pH 6.5 PBS buffer contained $3.96 \mathrm{~g} / \mathrm{L} \mathrm{EV}$ and $340 \mathrm{~g} / \mathrm{L}$ maltose was incubated at $45^{\circ} \mathrm{C}$ in combination with $20 \mu \mathrm{L}$ purified Agl2 or L145I/S272T. Squares: concentration of EVG catalyzed by L145I/S272T; circles: concentration of EVG catalyzed by Agl2; down triangles: concentration of EV in Agl2 reaction; up triangles: concentration of EV in L145I/S272T reaction.

\section{Supplementary Files}

This is a list of supplementary files associated with this preprint. Click to download.

- Supplementarymaterials.doc 